Alessandro Prato

Università di Siena

https://doi.org/10.18778/8220-506-0.04

\title{
LEOPARDI E LA LINGUA ITALIANA TRA TRADIZIONE E MODERNITÀ
}

Riassunto: Il saggio si propone di illustrare le idee di Leopardi sulla crisi della lingua e della cultura italiana del primo Ottocento, contrassegnata da un provincialismo intellettuale che non le ha permesso di partecipare in modo adeguato allo sviluppo delle scienze e della filosofia che ha caratterizzato l'Europa del XVIII secolo. Dallo Zibaldone al Discorso sopra lo stato presente dei costumi degli italiani viene ricostruita la critica lucida e disincantata dei problemi che affliggono l'Italia della Restaurazione: il divario tra lingua parlata e lingua scritta, tra letteratura e scienza e tra cultura alta e cultura popolare, la mancanza di senso civico e di coesione sociale, lo scarso sviluppo dell'opinione pubblica, l'arretratezza culturale, la mancata modernizzazione della lingua. La posizione critica di Leopardi ha anche un significato politico e sociale e presenta elementi di evidente attualità che sono ancora di grande interesse per i lettori del XXI secolo.

Parole chiave: linguaggio, sociolinguistica, filosofia politica, retorica.

\begin{abstract}
Leopardi and the Italian language between tradition and modernity. The essay aims to illustrate the Leopardi's ideas on the crisis of the Italian language and culture of the early nineteenth century, marked by an intellectual provincialism that did not allow it to participate adequately in the development of the sciences and philosophy that characterized Europe 18th century. The lucid and disenchanted criticism of the problems affecting Italy of the Restoration is reconstructed: the gap between spoken and written language, between literature and science and between high culture and popular culture, the lack of civic sense and social cohesion, the scarce development of public opinion, cultural backwardness, lack of modernization of the language. Leopardi's critical position also has political and social significance and presents elements of evident relevance which are still of great interest to the readers of the 21 st century.
\end{abstract}

Keywords: language, sociolinguistics, political philosophy, rhetoric. 
Il pensiero linguistico di Leopardi è contenuto in massima parte nello Zibaldone, soprattutto nei pensieri redatti tra il 1821 e il 1824, ma riflessioni di carattere sociolinguistico in cui la problematica linguistica è strettamente connessa a quella politica e sociale si ritrovano anche nel fondamentale Discorso sopra lo stato presente dei costumi degli italiani, scritto probabilmente nel 1824 e pubblicato postumo solo nel $1906^{1}$. Leopardi è consapevole sia della connessione stringente tra lingua, cultura e nazione, sia del condizionamento sociologico subito dalla lingua in base alla situazione politico-economica della capitale di un paese e del suo rapporto con il territorio. Come Vico, Leopardi è interessato alla storia dell'uomo e alle istituzioni, tra le quali la lingua ha un posto di rilievo perché la coscienza collettiva di una nazione si riverbera in essa $^{2}$ : come scrive nella lettera a Giordani del 13 luglio 1821, lo studio delle lingue richiede "tanta profondità di concetti quanta può capire nella mente umana, stante che la lingua, l'uomo e le nazioni per poco non sono la stessa cosa" (Leopardi 1969: 1123). Nell'ottica di Leopardi tutto ciò che è umano ha a che fare in qualche modo con la lingua, dalla letteratura, alla politica, alla storia nazionale e al sistema delle idee (D’Intino - Maccioni 2016: 125). La sua concezione materialista dell'uomo e delle sue facoltà conoscitive è collegata al rapporto di reciproca dipendenza tra il pensiero e il linguaggio:

Nelle parole si chiudono e quasi si legano le idee, come negli anelli le gemme, anzi si incarnano come l'anima nel corpo, facendo seco loro come una persona, in modo che le idee sono inseparabili dalle parole, e divise non sono più quelle, sfuggono all'intelletto e alla concezione, e non si ravvisano, come accadrebbe all'animo nostro disgiunto dal corpo $(\mathrm{Zib} .2584)^{3}$.

Lo Zibaldone è anche una preziosa risorsa per ricostruire la formazione di Leopardi e il complesso intreccio di letture e suggestioni che hanno alimentato la sua riflessione. Tra le fonti utilizzate spiccano tutti i testi fondativi della retorica, a conferma dell'interesse che questo ambito di studi ha sempre rappresentato per lui; anche la Poetica e la Retorica di Aristotele hanno svolto un ruolo centrale nella sua formazione, se Leopardi stesso, in una delle prime pagine del diario, ha menzionato Aristotele, il fondatore dell'arte del discorso, considerandolo uno di quegli autori "al quale volendo o non volendo senzavvedersene si ritorna" $(Z i b: 16)^{4}$. L'interesse per la retorica in Leopardi si manifesta in forme diverse

1 Utilizziamo l'edizione a cura di M. Dondero: Leopardi (1998).

2 È un tema centrale dell'ideario leopardiano a cui fanno riferimento Gensini (1998); Basile (2018); Bellomo (2018).

3 Si cita sempre la pagina dell'autografo riportata in tutte le edizioni.

4 Sull'interesse di Leopardi per la retorica mi permetto di rinviare a Prato (2019). 
e coinvolge molteplici ambiti sia storici, sia teorici; tra questi emerge con particolare evidenza il problema del rapporto tra lingua e la collettività.

Lo sviluppo delle scienze e del pensiero filosofico del XVIII secolo aveva portato alla conoscenza di nuove idee. La lingua italiana si trovava impreparata ad esprimere questi concetti nuovi, sia perché mancava dei vocaboli ad essi corrispondenti, sia perché non aveva una prosa agile e scorrevole adatta a esprimere e divulgare queste idee. Per compensare questa mancanza si erano adottati termini e frasi straniere, specialmente francesi, in maniera forse eccessiva, al punto da creare il pericolo di un nuovo bilinguismo ${ }^{5}$.

Sulla base di queste considerazioni la posizione di Leopardi rispetto al purismo è più complessa e sfumata di quella dei filosofi illuministi, anche se non mancano, naturalmente, molteplici aspetti di convergenza. Leopardi, infatti, riconosce che il purismo in parte era stato utile e necessario, nel suo intento di frenare la corruzione dell'italiano dovuta all'eccessiva adozione di vocaboli stranieri. Tuttavia, nella sua pretesa di ripristinare l'indole antica della lingua italiana, così com'era nella sua stagione d'oro (il Trecento), nell'ammirazione della tradizione letteraria più antiquata, nell'ostilità per l'illuminismo, il purismo è stato anche conservatore e nocivo in quanto espressione del "patriottismo reazionario, tradizionalista e antifrancese" (Timpanaro 1969: 65). Di tutto questo Leopardi è ben consapevole, infatti egli critica gli scrittori avversari dei puristi come Cesarotti che, sentendo la giusta necessità di rimodernare la lingua e la cultura italiana, avevano proposto però l'adozione dei modelli stranieri, senza tener conto della lunga e complessa tradizione italiana; anzi, spesso, senza neanche conoscerla. Allo stesso tempo, però, critica anche il purismo le cui idee in fatto di lingua gli sembrano segnate dalla pedanteria.

Anche Pietro Giordani, dopo un periodo di simpatia e adesione al purismo, ha finito per dare di questo movimento un giudizio fortemente negativo: "Il principio dell'età corrente mostrò un paralitico desiderio di rifarsi italiano; come se dal belletto e non dal sangue venisse l'aspetto di sanità: tutto finì prestamente in miserabil pedanteria di pochi” (Giordani 1854-63: XI, 165) ${ }^{6}$. Giordani non condivide del purismo neanche l'idea che la 'proprietà della parola fosse data una volta per tutte e coincidesse col suo valore etimologico o più anticamente attestato. Al contrario, egli sostiene che una parola può avere diversi e successivi significati tutti propri, cioè corrispondenti a un determinato stadio di sviluppo sociale e concettuale. Ci sembra interessante ricordare che Giordani, proprio in questo periodo, progettava di scrivere un'opera sulla Storia dello spirito pubblico d'Italia per seicento anni considerato nelle vicende della lingua.

5 Si veda a questo proposito Migliorini, Baldelli (1984: 214-244).

6 Sulla posizione di Giordani nella questione della lingua rimane fondamentale Timpanaro (1980: 107-122). 
Quest'opera, come del resto tante altre, è rimasta solo allo stato di abbozzo; se egli l'avesse completata, sicuramente avrebbe sostenuto posizioni analoghe a quelle di Leopardi sullo stato della lingua italiana e sui motivi della sua crisi. Negli appunti preparatori di questo lavoro emerge tuttavia la sua consapevolezza del nesso tra lingua, società e cultura:

La lingua la considero come uno specchio dove si riverberano, e donde poi si riflettono tutti i costumi, le passioni, i casi d'una nazione [...] Io voglio nelle variazioni della lingua trovare di mano in mano, la spia di tutte le variazioni, non solo delle rumorose notate nelle storie, ma delle più intime e inosservate, che accaddero alla nostra nazione. E considerando poi la lingua ridotta a stile, come una delle arti imitatrici, voglio colle variazioni nazionali esplicare i mutamenti successivi dello stile. Le guerre, il commercio, le sette filosofiche, le religiose, le mode, tutto altera la lingua e lo stile ne' popoli (ivi: II, 143).

Il panorama della situazione linguistica e culturale dell'Italia della Restaurazione è caratterizzato dal problema della costruzione di una lingua nazionale moderna che non aveva ancora trovato una soluzione soddisfacente, e dalla scissione tra lingua parlata e scritta che anche Manzoni considera molto importante, come dimostra la lettera a Fauriel del 9 febbraio 1806 in cui dice che "per nostra sventura, lo stato dell'Italia divisa in frammenti, la pigrizia e l'ignoranza hanno posta tanta distanza tra la lingua parlata e la scritta, che questa può dirsi quasi lingua morta" (Manzoni 1954-74: IX, 331). In questo contesto Leopardi assume una posizione originale, dettata dal suo personale modo di considerare le lingue, alternativa sia al purismo che ai suoi detrattori; a questo proposito risultano particolarmente interessanti i passi rubricati nell'indice autografo sotto la voce 'Letteratura e lingua italiana d'oggidì. La voce è accompagnata dalla breve e importante annotazione: "Trista condizione di un vero letterato in Italia. Gli bisogna fare all'Italia una lingua moderna. Considerazioni in questo proposito". In questi pensieri la mancanza di una lingua moderna italiana viene fatta derivare dalla crisi della cultura italiana, sia in riferimento alla letteratura che alla filosofia ${ }^{7}$ :

Come noi non abbiamo se non letteratura antica, e come la lingua illustre e propria ad essere scritta non è mai scompagnata dalla letteratura, e segue sempre le vicende di questa, e dove questa manca e s'arresta, manca essa pure e si ferma; così fermata tra noi la letteratura fermossi anche la lingua, e siccome della letteratura, così pur della lingua illustre si deve dire, che noi

7 Per la stretta correlazione tra la letteratura e la lingua vedi ad esempio Zib. 1093. Per un approccio socio-linguistico al "problema" dell'italiano vedi Bollati (1983: 44 ss). 
non ne abbiamo se non antica. Sono oggimai più di centocinquant'anni che l'Italia né crea, né coltiva per sé verun genere di letteratura, perché in niun genere ha prodotto scrittori originali dentro questo tempo ( $\mathrm{Zib}$. 3319).

Quel progetto riformista che gli intellettuali illuministi avevano promosso con tanto entusiasmo e determinazione, non aveva ancora trovato attuazione, e, anzi, negli anni in cui scrive Leopardi, si era forse definitivamente arenato, perché

fra queste generali vicende e questo progresso della letteratura, l'Italia, come di sopra dissi, nulla ha fatto per sé. Gli scrittori alquanto originali che essa ha prodotti in questo tempo, gli scrittori che possono meritare il nome di moderni, non sono stati sufficienti, né per originalità né per numero, a darle una lingua nazionale moderna, nello stesso modo che ei non sono stati sufficienti a fare ch'ella avesse una letteratura moderna nazionale (Zib. 3321-3322).

Mancando quindi la letteratura, la lingua è rimasta quella illustre del tempo antico, che non è idonea a esprimere nuove idee. Per uscire da questa situazione Leopardi propone allora in un certo senso di rimettere in cammino la lingua italiana, di ridarle nuova linfa culturale e vigore, tenendo conto però della sua ricca, variegata e inestimabile storia:

quanto è facile il continuare a una nazione la sua lingua illustre insieme colla sua letteratura, tanto è difficile, interrotta per lungo spazio la letteratura, e dovendo quasi ricrearla, riannodare la lingua a lei conveniente colla già antiquata lingua illustre della nazione, colla lingua che fu propria della letteratura prima che questa fosse totalmente interrotta ( $\mathrm{Zib}$. 3323).

Da questo scaturisce la singolare condizione in cui si trova la lingua e, di conseguenza, lo scrittore in Italia: avere a disposizione un formidabile strumento espressivo, che, tuttavia, per ragioni storiche, non è in sé utilizzabile dallo scrittore che vuole essere moderno e che desidera confrontarsi con la cultura europea più avanzata:

Noi abbiamo una lingua; antica bensì, ma ricchissima, vastissima, bellissima, potentissima, insomma colma d'ogni sorta di pregi [...] questa lingua italiana che noi ci troviamo, supera di ricchezza, di potenza, di varietà tutte le lingue moderne, salvo forse la tedesca; di bellezza avanza d'assai tutte queste lingue senza eccezione né dubbio alcuno (...) tale si è la lingua italiana per sé ed intrinsecamente. Ma ella è antica; cosa estrinseca; ed essendo antica non basta, né si adatta, tal quale ella è a chi vuole scriver cose moderne in maniera moderna (Zib. 3326-3327). 
La lingua antica così comè non può essere utilizzata oggi, su questo Leopardi non cambia posizione: "né mai in una lingua antica si potranno scriver cose moderne né scriverle modernamente" ( $Z i b .3337)$. La situazione in cui si viene a trovare lo scrittore moderno è quindi molto difficile:

si consideri le grandissime difficoltà ed ostacoli che si attraversano, le angustie che stringono, la vera infelicità della condizione in cui si trova oggidì l'italiano che aspiri ad esser scrittore classico, cioè pensare originalmente, dir cose proprie del tempo, dirle in modo proprio del tempo, e perfettamente adoperare la sua lingua, senza le quali condizioni, e una sola che ne manchi, non si può mai né pretendere giustamente, né ragionevolmente sperare l'immortalità letteraria $(\mathrm{Zib} .3327)$.

E, ancora, nello stesso giro di pensieri leggiamo che

certo è veramente dura e deplorabile oggidì la condizione dell'italiano il quale avesse nella sua mente cose degne d'esser scritte e convenienti à nostri tempi, perocch'egli, anche volendo usare la maggior semplicità del mondo, non avrebbe una lingua naturale in cui scrivere (come l'hanno i francesi ecc. atta a potervi subito scrivere, com'ei l'abbiano competentemente coltivata e studiata), né il modo di bene esprimere i suoi concetti gli correrebbe mai alla penna spontaneo, ma converrebbe ch'egli si fabbricasse l'istrumento con cui significar le sue idee ( $Z i b$. 3321).

Questo passo è molto interessante perché contiene l'autoritratto dello scrittore moderno che si accingeva proprio in quel periodo a scrivere le Operette morali, in cui questo progetto linguistico trova una felicissima attuazione. La ricerca di un adeguato strumento linguistico è collegata sempre alla scelta dei contenuti da esprimere, ai sistemi di idee che le parole rappresentano e fanno circolare. La posizione di Leopardi è piuttosto isolata nel dibattito del tempo dove, dopo l'esperienza riformatrice, la discussione sulla questione della lingua tendeva a ripresentarsi solo come ricerca linguistica fine a sé stessa, sterile e inutile, o come espressione di una retorica priva dello spessore culturale e molto distante, quindi, dai modelli di eloquenza che erano operanti a metà Settecento, per esempio, in Genovesi e Beccaria. Ed è proprio questa riproposizione a essere stigmatizzata da Leopardi che non si riconosce in quegli scrittori che si preoccupano solo dei fatti linguistici, senza impegnarsi in alcun modo; la loro produzione non poteva risultargli più estranea:

la loro scrittura non manifesta alcun pensiero degno di nota, il numero e il valore di quelle ombre di filosofi che ha veduto fin qui l'Italia, va pur sempre notabilmente scemando, e sempre per lo contrario crescendo, non il 
valore, ma il numero di quelli che pretendono e aspirano a scrivere il buon italiano; onde l'Italia è quasi tutta rivolta di nuovo alla sua antica lingua, e di pensieri oramai nulla più pensa né cura né richiede; propriamente nulla (Zib. 335-336).

Anche il dissenso che Leopardi manifesta nei confronti della cultura del tempo è molto forte: in un pensiero del 1821 scrive che "Tutte le opere letterarie italiane d'oggidì sono inanimate, esangui, senza moto, senza calore, senza vita" ( $Z i b .725)$; la frequentazione dell'ambiente romano tra il 1822 e il 1823 gli causa una profonda delusione, come testimoniano le lettere scritte a Giordani, ad esempio in quella del 1 febbraio 1823 dice che "La letteratura romana, come tu sai benissimo, è così misera, vile, stolta, nulla $[\ldots]$ questi miserabili letterati mi disgustano della letteratura" (Leopardi 1969: 1146). Ancora nella lettera al padre del 9 dicembre 1822 Leopardi scrive:

quanto ai letterati dei quali Ella mi domanda, io n'ho veramente conosciuto pochi, e questi pochi m'hanno tolto la voglia di conoscerne altri $[\ldots]$ secondo loro il sommo della sapienza umana, anzi la sola e vera scienza dell'uomo è l'antiquaria. Non ho ancora potuto conoscere un letterato romano che intenda sotto il nome di letteratura altro che l'archeologia. Filosofia, morale, politica, scienza del cuore umano, eloquenza, poesia, filologia, tutto ciò è straniero a Roma (ivi: 1133);

nella lettera al fratello Carlo del 14 dicembre 1822 il tono è anche più aspro e drastico:

Della letteratura non so che mi vi dire. Orrori e poi orrori. I più santi nomi profanati, le più insigne sciocchezze levate al cielo, i migliori spiriti di questo secolo calpestati come inferiori al minimo letterato di Roma, la filosofia disprezzata come studio da fanciulli, il genio, l'immaginazione e il sentimento, nomi incogniti e forestieri ai poeti e alle poetesse di professione (ivi: 1135).

La classe degli intellettuali italiani presenti a quel tempo a Roma appare a Leopardi meschina e di scarso valore, tutta imprigionata nel proprio cinismo e nella vacuità delle azioni che intraprende. E di questa classe era ai suoi occhi un degno rappresentante il noto erudito Francesco Cancellieri, di cui in una lettera sempre al fratello Carlo, scritta nello stesso periodo, disegna un proverbiale ritratto: "ieri fui da Cancellieri, il qual è un coglione, un fiume di ciarle, il più noioso e disperante uomo della terra; parla di cose assurdamente frivole col massimo interesse, di cose somme colla maggiore freddezza possibile" (ivi: 1130). A Vieusseux che gli propone di collaborare all'Antologia con degli 
articoli sulle novità intellettuali del momento, Leopardi risponde esprimendo tutto il suo disgusto per la vita culturale italiana di allora, della quale pensa che non vale neanche la pena di occuparsi: "i giornali stranieri sono utili quando annunziano, perché hanno sempre opere degne da analizzare, o cose che meritano di essere riferite. Ma i libri che oggi si pubblicano in Italia non sono che sciocchezze, barbarie, rancidumi, copie e ripetizioni" (ivi: 1178)

Da cosa dipende questo ritardo civile e culturale dell' Italia? Quale è la causa della mancanza di società in Italia? Perché l'opinione pubblica e il pensiero critico sono molto limitati e poco organizzati rispetto agli altri paesi europei? La risposta che Leopardi esprime sia nei pensieri dello Zibaldone, sia nel Discorso sopra lo stato presente dei costumi degli italiani, è molto lucida: perché l'Italia - a differenza degli altri paesi e anche della Germania, che pure è politicamente divisa - "non è neppure una nazione, né una patria" ( $\mathrm{Zib}$. 2065); in Italia

la forma di governo è tale che la nazione non v'ha alcuna parte, gli affari sono in man di pochissimi e separatissimi dal resto de' nazionali tutto si passa senza pur venire a notizia della nazione, sicché la politica è affatto ignota ed aliena alla nazione medesima, la libertà di ciascheduno è così circoscritta che ciascheduno è ben poco in grado di determinar la sua sorte, e di governarsi, ma quanto più si può è governato veramente da altrui, e ciò non dalla nazione, non dal comune, non ciascuno da tutti, ma tutti da uno o da pochissimi particolari, e il pubblico, per così dire da' privati (Zib.3858-3860).

Alla pagina successiva Leopardi riprende l'argomento osservando che ciò che caratterizza la situazione politico-culturale del suo tempo è soprattutto l'assenza di

letteratura, d'industria, di società, di arti, di genio, di coltura, di grandi ingegni, di facoltà inventiva, d'originalità, di passioni grandi, vive, utili o belle e splendide, d'ogni vantaggio sociale, di grandi fatti e quindi di grandi scritti, inazione torpore ( $\mathrm{Zib} .3861)$.

La mancanza di una lingua e di una società moderna in Italia dipende dalla mancanza di una nazione, dalla sua nullità politica e militare, dal fatto che è priva di una capitale, una letteratura, un teatro, una conversazione sociale, cioè di quelle istituzioni che assicurano una uniformità di opinioni e di costumi che è poi alla base della coesione sociale:

8 Sul difficile rapporto con Vieusseux restano fondamentali le osservazioni di Timpanaro (1982: 164-167). Si veda anche Gensini (1998). 
Lascio stare che la nazione non avendo centro, non havvi veramente un pubblico italiano; lascio stare la mancanza di teatro nazionale, e quella della letteratura veramente nazionale e moderna, la quale presso l'altre nazioni, massime in questi ultimi tempi è un grandissimo mezzo e fonte di conformità di opinioni, gusti, costumi, maniere, caratteri individuali, non solo dentro i limiti della nazione stessa, ma tra più nazioni eziandio rispettivamente. Queste seconde mancanze sono conseguenze necessarie di quella prima, cioè della mancanza di un centro, e di altre molte cagioni. Ma lasciando tutte queste e quelle, e restringendoci alla sola mancanza di società questa opera naturalmente che in Italia non havvi una maniera, un tuono italiano determinato (Leopardi 1998: 56-57).

Per questa ragione gli italiani non possono essere cittadini, ma soltanto individui, ognuno dei quali fa "tuono e maniera da sé" (ivi: 57$)^{9}$ dato che "non v'è tuono di società che possa dirsi italiano" ( $Z i b .3546)$. Ecco perché gli italiani non hanno quell'orgoglio nazionale che, invece, è ben presente agli inglesi e ai francesi e che costituisce una grande forza per l'unità e la moralità di una nazione. Mancando lo schermo e il collante della società, primeggia sullo spirito pubblico italiano il cinismo, l'indifferenza se non il disprezzo verso gli altri, una sorta di conflitto sociale potenziale sempre pronto a esplodere alla prima occasione:

Quelli che credono superiore a tutte per cinismo la nazione francese, s'ingannano. Niuna vince né uguaglia in ciò l'italiana. Essa unisce la vivacità naturale (maggiore assai di quella de' Francesi) all'indifferenza acquisita verso ogni cosa e al poco riguardo verso gli altri cagionato dalla mancanza di società, che non li fa curar gran fatto della stima e de' riguardi altrui (Leopardi 1998: 65-66).

Questa visione così critica e disincantata della società italiana ritorna anche in altri due autori molto diversi tra loro con cui Leopardi a questo proposito presenta una singolare convergenza di pensiero. Il primo è Shelley che durante il soggiorno a Napoli nel 1818 scrive che

Ci sono due Italie; una costituita dalla terra verde, dal mare trasparente, dalle possenti rovine dei tempi antichi [... l'altra consiste degli italiani di oggi, delle loro opere e dei loro costumi. L'una è la più sublime e leggiadra visione che possa essere concepita dall'immaginazione umana; l'altra la più degradata, disgustosa e odiosa (Shelley 1996: 1144).

9 Vedi anche Aloisi (2014: 174-177), oltre a Prato (2012: 132-133). 
Il secondo è Gioberti, il quale nel suo Del primato morale e civile degli italiani (1842-1843), trattando temi analoghi a quelli che abbiamo incontrato nei testi leopardiani, ammette che il popolo italiano non esiste nella realtà, anticipando la frase molto nota e citata dopo l'unificazione che dice "fatta l'Italia bisogna fare gli italiani", attribuita spesso a Massimo D’Azeglio, ma che appartiene invece al meno noto Ferdinando Martini, deputato dal 1870 e più volte ministro (Soldani - Turi 1993: I, 17):

il popolo italiano è un desiderio e non un fatto, un presupposto e non una realtà, un nome e non una cosa, e non so pur se si trovi nel nostro vocabolario. V'ha bensì un'Italia e una stirpe italiana congiunti di sangue, di religione, di lingua scritta ed illustre; ma divisa di governi, di leggi, d'instituti, di favella popolare, di costumi, di affetti, di consuetudini (Gioberti 1925: I, 92-93).

La diagnosi di Leopardi della società italiana e delle forze politiche e culturali che governano l'opinione pubblica ricalca quel nesso tra letteratura e lingua, collegato a sua volta a quello, altrettanto centrale, tra nazione e lingua, cui abbiamo già fatto riferimento e che si risolve nella reciproca determinazione fra condizioni linguistiche, condizioni politiche e forme della produzione culturale, il cui intreccio può ricordare l'impostazione con cui Gramsci avrebbe considerato molto tempo dopo la questione della lingua ${ }^{10}$. Nel suo vigore polemico e nel suo profondo pessimismo, l'analisi politico-sociale elaborata da Leopardi colpisce per la sua manifesta attualità: ancora oggi possiamo osservare infatti quanto nel nostro paese il problema della mancanza di senso civico e di coesione sociale sia lontano dall'avere trovato una adeguata soluzione. E proprio su questo terreno si misura una interessante convergenza con le idee e la posizione di Manzoni, l'altro grande protagonista della letteratura e della linguistica ottocentesca; pur partendo da convinzioni molto diverse, la forza di penetrazione della loro critica raggiunge risultati analoghi ${ }^{11}$. Entrambi, come aveva osservato acutamente Sciascia (1983: 99), tendono a delineare un disperato e autentico ritratto dell'Italia, che è per molti aspetti ancora l'Italia di oggi, e sul quale vale ancora la pena di riflettere.

\section{Bibliografia}

Aloisi, Alessandra (2014). Desiderio e assuefazione. Studio sul pensiero di Leopardi, Pisa, Edizioni ETS.

Aloisi, Alessandra (2018). “La filosofia”, [in] Franco D’Intino e Massimo Natale (a c. di), Leopardi, Roma, Carocci, pp. 101-123.

10 Rimandiamo a Gensini (1998: XLIX-L).

11 Come mette ben in evidenza Dotti (1993: 5-6). 
Basile, Grazia. (2018). "La linguistica”, [in] Franco D’Intino e Massimo Natale (a c. di), Leopardi, Roma, Carocci, pp. 167-180.

Bellomo, L. (2018). "La lingua e lo stile”, [in] Franco D’Intino e Massimo Natale (a c. di), Leopardi, Roma, Carocci, pp. 201-225.

Bollati, Giulio (1983). L'Italiano, Torino, Einaudi.

D’Intino, Franco e Maccioni, Luca (2016). Leopardi: guida allo Zibaldone, Roma, Carocci.

Dotti, Ugo (1993). Il savio e il ribelle. Manzoni e Leopardi, Roma, Editori Riuniti.

Gensini, Stefano (1998). “Leopardi «filosofo linguista italiano»”, [in] Leopardi 1998a, pp. XLV-LIII.

Gensini, Stefano e Prato, Alessandro (a c. di) (2019). I segni fra teoria e storia per Giovanni Manetti, Pisa, Edizioni ETS.

Gioberti, Vincenzo (1925). Del primato morale e civile degli italiani, a c. di Giuseppe Balsamo Crivelli, Torino, Utet.

Giordani, Pietro (1854-1863). Opere, a c. di Antonio Gussalli, Milano, Borroni \& Scotti.

Leopardi, Giacomo (1969). Tutte le opere, a c. di Walter Binni e Enrico Ghidetti, Firenze, Sansoni.

Leopardi, Giacomo (1991). Zibaldone, a c. di Vincenzo. Placella, Milano, Garzanti.

Leopardi, Giacomo (1998). Discorso sopra lo stato presente dei costumi degli italiani, Milano, BUR.

Leopardi, Giacomo (1998a). La varietà delle lingue, a c. di Stefano Gensini con la collaborazione di Alessandro Prato, Firenze, La Nuova Italia.

Manzoni, Alessandro (1954-1974). Tutte le opere, a c. di Alberto Chiari e Fausto Ghisalberti, Milano, Mondadori.

Migliorini, Bruno e Baldelli, Ignazio (1983). Breve storia della lingua italiana, Firenze, Sansoni. Prato, Alessandro (2012). La retorica. Forme e finalità del discorso persuasivo, Pisa, Edizioni ETS.

Prato, Alessandro (2019). “ «Cogliere le somiglianze nella diversità delle cose»: sul concetto di metafora in Leopardi", [in] Stefano Gensini e Alessandro Prato, (a c. di) I segni fra teoria e storia per Giovanni Manetti, Pisa, Edizioni ETS, pp. 119-132.

Sciascia, Leonardo (1983). Cruciverba, Torino, Einaudi.

Shelley, Percy Bisshe (1996). Opere, a c. di Francesco Rognoni, Milano, Mondadori.

Soldani, Simonetta e Turi, Gabriele (a c. di) (1993). Fare gli italiani. Scuola e cultura nell'Italia contemporanea, Bologna, Il Mulino.

Timpanaro, Sebastiano (1969). Classicismo e illuminismo nell'Ottocento italiano, Pisa, NistriLischi.

Timpanaro, Sebastiano (1980). Aspetti e figure della cultura ottocentesca, Pisa, Nistri-Lischi.

Timpanaro, Sebastiano (1982). Antileopardiani e neomoderati nella sinistra italiana, Pisa, Edizioni ETS. 